\title{
No Refund or Full Refund: When Should a Fashion Brand Offer Full Refund Consumer Return Service for Mass Customization Products?
}

\author{
Tsan-Ming Choi, Na Liu, Shuyun Ren, and Chi-Leung Hui \\ Institute of Textiles and Clothing, Faculty of Applied Science and Textiles, The Hong Kong Polytechnic University, \\ Hung Hom, Kowloon, Hong Kong \\ Correspondence should be addressed to Tsan-Ming Choi; jason.choi@polyu.edu.hk
}

Received 7 December 2012; Revised 5 February 2013; Accepted 6 February 2013

Academic Editor: Pui-Sze Chow

Copyright (C) 2013 Tsan-Ming Choi et al. This is an open access article distributed under the Creative Commons Attribution License, which permits unrestricted use, distribution, and reproduction in any medium, provided the original work is properly cited.

\begin{abstract}
We analytically explore in this paper the consumer return policy under fashion mass customization (MC) program. To be specific, we model the stochastic fashion MC program with the consideration of consumer demand uncertainty. If a consumer return policy is implemented, we further consider return uncertainty. By modeling the optimization objective of the risk averse MC fashion brand via a mean-variance approach, we derive the closed-form optimal solution under each case. We then conduct both analytical and numerical sensitivity analyses. For the scenario with full refund and return, we reveal the analytical conditions under which the optimal retail price and the optimal number of options available for customization (called the "optimal modularity level") vary monotonically with respect to the salvage value and the return service charge. For the scenario when there is no refund and return, we show that the optimal retail price and the optimal modularity level are decreasing in the MC fashion brand's degree of risk aversion, the demand uncertainty, and the price-demand sensitivity coefficient. In addition, our numerical analysis indicates that whether the risk averse MC fashion brand would prefer offering consumer return with full refund to no return depends heavily on the demand-return correlation (DRC) parameter.
\end{abstract}

\section{Introduction}

Mass customization (MC) is an industrial practice which helps to manufacture customized products to satisfy consumers' needs with a cost close to mass production [1-7] in a timely manner [8-10]. In the fashion industry, as driven by the consumer needs [11-13], the advances of production and information technology [14-21] and the increasingly competitive market situation [22-25], MC has developed to be a very popular industrial practice $[17,26]$ and a lot of fashion retail brands and fashion retailers are implementing it. In fact, we can find MC in nearly all kinds of wellestablished fashion brands, from high-end luxury brands (Hermes, LV, etc.) to mass-market labels (Adidas, Nike, Puma, Brooks Brothers, etc.) (see $[8,27]$ for more cases and empirical details). However, fashion brands offering MC services face some challenges, and it is known that big name retailers/brands like Levi's and Lands' End have already terminated their MC programs for different reasons.
In recent years, there are more and more analytical studies on fashion MC and some of them concern about consumer returns. In particular, Liu et al. [28] develop a three-dimensional optimization problem and analytically derive the optimal decisions on retail price [29], modularity level [30-32], and refund rate (under consumer return [33]). From both analytical and numerical analyses, they obtain several important findings and insights. Among others, they argue that the degree of risk aversion of the fashion MC service provider is an influential factor affecting the respective optimal decisions. In addition, Choi [34] studies the optimal return service charging policy for fashion retailers offering MC. He develops the closed-form analytical conditions under which it is optimal for the fashion MC service provider to provide free return service charge to consumers.

In the fashion industry, the adoption of consumer return under the MC service program is very polarized. Actually, we find that the fashion MC brands either simply do not 
allow any consumer return or they would allow full refund in the return policy. For example, the international brand Nike is implementing MC and it allows consumer return with full refund for its NIKEiD products. (p.s.: Nike makes it clear on its website that under its NIKEiD MC program, any consumer can return the MC products (with any reasons) within 30 days of purchase if they want to.) However, many other similar sporty fashion brands do not allow return for MC products (except for those with manufacturing defects or quality problems).

In light of the above industrial observation and based on the prior studies in the literature, we examine in this paper the optimal retail pricing and modularity level decisions for a risk averse MC fashion brand (p.s.: an MC fashion brand is a fashion brand which is offering MC.) for both scenarios with and without consumer return. We study the optimal pricing and modularity level decisions under each scenario. We analytically reveal that a linear relationship exists between the optimal price and the optimal level of modularity under both scenarios. We also show analytically how the optimal pricing and level of modularity decisions relate to some important model parameters. After that, we explore the factors which will affect the risk averse MC fashion brand's decision on when to offer consumer return with full refund. We find that whether it is optimal for the risk averse MC fashion brand to offer consumer return with full refund or no return depends critically on the demand return correlation coefficient.

The rest of this paper is organized as follows. We review the related literature concisely in Section 2 . We present the analytical models in Section 3. We derive the optimal decisions in Section 4. We conduct numerical sensitivity analysis in Section 5. We conclude and discuss future research directions in Section 6.

\section{Literature Review}

MC is an important make-to-order/build-to-order [32, 35] business strategy widely adopted in fashion retailing. In fact, MC helps consumers to develop customized products [36] and participate in the codesign process [37] which is a known means of cocreating value $[38,39]$. It is also a popular topic in operations management (see [40] for a relatively recent review) and both empirical and analytical studies are reported in recent years. For example, Peters and Saidin [16] explore via a case study approach the challenges of MC implementation. They study the critical factors which drive the service provider to adopt MC. They discuss the challenges behind the MC implementation in the service sector. They further propose a novel framework which can help the service provider to offer MC. Gu et al. [41] develop an optimization model to describe the implementation of $\mathrm{MC}$ as a gradual process. They derive two optimization methods to find the optimal customization quantity. They indicate that their proposed methods can successfully reduce the amount of customized items in every part of the overall production process and hence enhances the efficiency of MC. Helms et al. [42] examine e-commerce and knowledge management and identify how they can support
MC. They find that e-commerce provides capabilities for the MC service provider to reach global market and helps in learning about customer preferences. They reveal that knowledge management can help manage the intellectual capital. They argue that both e-commerce and knowledge management can significantly improve the implementation of MC. Bock [43] studies the pros and cons on employing off-shore and near-shore production strategies under MC. He proposes a mixed-model assembly line balancing approach to provide a direct comparison of the estimated variable manufacturing costs by generating a location-based line layout for all competing locations. He runs several tests with various location configurations and identifies the factors which affect the optimal sourcing and production strategy for the MC company. Brun and Zorzini [44] investigate the relationships between postponement and modularization under MC. They examine the industrial cases in Italy with a case study approach. They reveal that product/process customization and product/process complexity are critical factors which affect the postponement and modularization relationship. Yao and Liu [45] study an interesting problem on how companies handle the probable contradiction between scale production effect and customized demand under MC. They develop a dynamic multiobjective optimization model and derive effective algorithm to solve it. They test their proposed algorithm by simulation and show that their proposed algorithm can enhance the scheduling efficiency under the MC program. Most recently, motivated by a typical MC production company that has an inefficient scheduling operations problem, Zhong et al. [46] examine the applicability of an RFID-enabled manufacturing execution system (MES) for MC. In their proposed MES, the RFID-based devices are deployed on the shop floor to keep track of the production data. As a result, they find that both operational planning and scheduling decisions become enhanced. They conduct a case study and conclude that their proposed MES is effective.

In the domain of fashion apparel, MC has also been extensively studied. For example, Dong et al. [1] discuss how MC can be implemented and integrated with the mass production line to achieve high quality and low price fashion products. Ulrich et al. [37] study the consumer-to-design scheme of fashion products under MC and generate a number of interesting insights. Yeung and Choi [27] explore the implementation of MC in the Hong Kong apparel industry. They identify a few barriers which hinder the development of fashion MC in markets like Hong Kong. Choi et al. [47] conduct a mean-variance analysis of the fashion MC program with consumer return. Based on the assumption that the consumer demand function is independent of the return service charging policy, they derive a couple of counterintuitive findings. Liu et al. [28] study the optimal retail pricing, refund, and modularity decision making problem for a fashion mass customization system. They obtain several important insights via extensive sensitivity analysis.

Based on the above reviewed literature, this paper conducts an analytical research on MC program with a risk averse fashion brand under the mean-variance framework [48, 49]. This paper is related to $[28,32,47]$ because they all consider optimal decisions related to retail pricing and modularity. 
However, this paper is different from $[28,32]$ in which the demand and return uncertainty models are different and this paper focuses on examining the polarized strategic decisions on allowing consumer return with full refund or disallowing return at all whereas $[28,32]$ focus on the optimal operational decisions on price, modularity, and refund rate. This paper is also different from [47] because we argue that demand must relate to the return service charge whereas [47] assumes consumer demand independent of return service charge. Finally, this paper is also different from [34] because these two papers have totally different optimal decisions and the research objectives are also different.

\section{Analytical Models}

In this paper, we consider a risk averse MC fashion brand which sells an MC fashion product directly to consumers via an online platform (e.g., the MC retailing website). The whole process is a make-to-order type and the fashion brand will start producing the product after receiving the order from the consumer. In this paper, we consider the situation that the MC fashion brand makes a few important decisions, namely the retail price of the MC product $(p)$, the number of options available for customization (we call it the level of modularity of the product $(m)$ ) (notice that this product modularity is different from the production process modularity concept commonly explored in the literature [50]), and whether to allow consumer return with full refund or no return. Notice that the "consumer return scheme" here refers to the product return from consumers who are unhappy with the purchase with any reason (probably within some reasonable time, such as one month), but it is not related to the situations with manufacturing mistakes, defects, or other product quality problems. As widely observed from the industrial practice in the fashion apparel industry, the MC fashion brands are adopting very polarized measure on consumer return: they either disallow return (i.e., no return, no refund) or accept return with full refund. Based on this industrial practice, we first represent the amount of refund under the consumer return policy by $r$. Thus, a full refund under the consumer return scheme implies $r=p$, and the no return scheme has $r=0$.

Following [28, 32], we construct the following analytical models in two separate scenarios, namely "full refund under consumer return" and "no consumer return." The notation employed in this paper basically follows the literature $[28,32,34]$.

\subsection{Full Refund under Consumer Return}

Demand function: $\widetilde{D}_{1}=\alpha-\beta p+\gamma r+\delta m+\widetilde{\varepsilon}_{D},(\beta>$ $\gamma$ ); (p.s.: notice that this price dependent function is very commonly employed in the literature, see, e.g., $[25,51]$.)

Return quantity function: $\widetilde{R}=\phi+\psi r+\widetilde{\varepsilon}_{R}$;

Salvage value: $S(m)=s+v m$;

Modularity cost: $C(m)=(1 / 2) \theta m^{2}$.
Since under the consumer return scheme, we assume a full refund will be granted for each return, we have the rate of refund $r=p$. Thus

$$
\widetilde{D}_{1}=\alpha-\beta p+\gamma(p-l)+\delta m+\widetilde{\varepsilon}_{D}, \quad(\beta>\gamma)
$$

(p.s.: we use subscripts 1 and 2 to represent the scenarios "with consumer return and full refund" and "no return," respectively.) $\widetilde{R}=\phi+\psi(p-l)+\widetilde{\varepsilon}_{R}, l<p$ is the service charge.

The profit function is hence expressed as follows:

$$
\begin{aligned}
P_{1}= & p\left(\alpha-\beta p+\gamma(p-l)+\delta m+\widetilde{\varepsilon}_{D}\right) \\
& +(S(m)-l)\left(\phi+\psi(p-l)+\widetilde{\varepsilon}_{R}\right)-\frac{1}{2} \theta m^{2} .
\end{aligned}
$$

We consider the scenario when the demand function and return quantity are correlated with a coefficient of correlation of $\rho$. Thus, the expected profit and the variance of profit are given as follows:

$$
\begin{aligned}
E\left[P_{1}\right]= & p[\alpha-\beta p+\gamma(p-l)+\delta m] \\
& +(s+v m-l)[\phi+\psi(p-l)]-\frac{1}{2} \theta m^{2} \\
V\left[P_{1}\right]= & p^{2} \sigma_{D}^{2}+(s+v m-l)^{2} \sigma_{R}^{2} \\
& +2 \rho \sigma_{R} \sigma_{D} p(s+v m-l) .
\end{aligned}
$$

Define in the following the mean-variance (MV) objective function [28, 52-57] of the MC fashion brand, where $k$ represents the degree of risk aversion for the $\mathrm{MC}$ fashion brand:

$$
U_{1}=E\left[P_{1}\right]-k V\left[P_{1}\right]
$$

In order to examine whether $U_{1}$ is concave, we construct the Hessian matrix as follows:

$$
\begin{aligned}
H_{U_{1}}= & \left|\begin{array}{ll}
\frac{\partial^{2} U_{1}}{\partial m^{2}} & \frac{\partial^{2} U_{1}}{\partial m \partial p} \\
\frac{\partial^{2} U_{1}}{\partial p \partial m} & \frac{\partial^{2} U_{1}}{\partial p^{2}}
\end{array}\right| \\
= & \left|\begin{array}{cc}
-\theta-2 k v^{2} \sigma_{R}^{2} & \delta+v \psi-2 k v \rho \sigma_{D} \sigma_{R} \\
\delta+v \psi-2 k v \rho \sigma_{D} \sigma_{R} & -2 \beta+2 \gamma-2 k \sigma_{D}^{2}
\end{array}\right| \\
= & \left(2 \beta-2 \gamma+2 k \sigma_{D}^{2}\right)\left(\theta+2 k v^{2} \sigma_{R}^{2}\right) \\
& -\left(\delta+v \psi-2 k v \rho \sigma_{D} \sigma_{R}\right)^{2}
\end{aligned}
$$


TABLE 1

\begin{tabular}{lcccccccccccccc}
\hline Parameter & $\alpha$ & $\beta$ & $\gamma$ & $\theta$ & $\delta$ & $\phi$ & $\varphi$ & $v$ & $s$ & $l$ & $k$ & $\rho$ & $\sigma_{D}$ & $\sigma_{R}$ \\
\hline Value & 2000 & 30 & 2 & 30 & 10 & 20 & 8 & 0.2 & 1 & 0.8 & 0.02 & 0.3 & 100 & 20 \\
\hline
\end{tabular}

$$
\begin{aligned}
= & 2 \theta(\beta-\gamma)+2 k \theta \sigma_{D}^{2}+4 k \rho \sigma_{D} \sigma_{R}\left(v \delta+v^{2} \psi\right) \\
& +4 k v^{2} \sigma_{R}^{2}(\beta-\gamma)+4 k^{2} v^{2} \sigma_{D}^{2} \sigma_{R}^{2}\left(1-\rho^{2}\right)-(\delta+v \psi)^{2}>0 .
\end{aligned}
$$

By checking the Hessian matrix, we can see that $U_{1}$ will be a concave function of $p$ and $m$ if and only if (5) holds.

3.2. No Consumer Return. For the scenario without consumer return, the model is much simpler because there will be no return and no salvage value of the returned product. As such, the demand function, the modularity cost, and the profit function are derived as follows.

$$
\begin{aligned}
& \text { Demand function: } \widetilde{D}_{2}=\alpha-\beta p+\delta m+\widetilde{\varepsilon}_{D} \text {. } \\
& \text { Modularity cost: } C=(1 / 2) \theta m^{2} .
\end{aligned}
$$

The profit function is:

$$
P_{2}=p\left(\alpha-\beta p+\delta m+\widetilde{\varepsilon}_{D}\right)-\frac{1}{2} \theta m^{2}
$$

The expected profit and variance of profit are hence given as follows:

$$
\begin{gathered}
E\left[P_{2}\right]=p(\alpha-\beta p+\delta m)-\frac{1}{2} \theta m^{2} \\
V\left[P_{2}\right]=p^{2} \sigma_{D}^{2}
\end{gathered}
$$

Similarly, we construct the MV objective function as follows:

$$
U_{2}=E\left[P_{2}\right]-k V\left[P_{2}\right]
$$

By checking the Hessian matrix, we can see that $U_{2}$ is a concave function of $p$ and $m$ if and only if (9) is satisfied as follows:

$$
\begin{aligned}
H_{U_{2}} & =\left|\begin{array}{ll}
\frac{\partial^{2} U_{2}}{\partial m^{2}} & \frac{\partial^{2} U_{2}}{\partial m \partial p} \\
\frac{\partial^{2} U_{2}}{\partial p \partial m} & \frac{\partial^{2} U_{2}}{\partial p^{2}}
\end{array}\right|=\left|\begin{array}{cc}
-\theta & \delta \\
\delta & -2 \beta-2 k \sigma_{D}^{2}
\end{array}\right| \\
& =2 \theta\left(\beta+k \sigma_{D}^{2}\right)-\delta^{2}>0 .
\end{aligned}
$$

\section{Optimal Pricing and Modularity Level Decisions}

For a notational purpose, we define the following:

$$
\begin{aligned}
& A_{1}= \theta(\alpha-l \gamma)-\theta \psi(l-s)+v(\delta+v \psi)(\phi-l \psi), \\
& B_{1}=2 \rho \sigma_{D} \sigma_{R}\left(l \theta-s \theta-v^{2} \phi+l v^{2} \psi\right)+2 \sigma_{R}^{2}\left(v^{2} \alpha-l v^{2} \gamma+l v \delta-s v \delta\right), \\
& G_{1}=(\delta+v \psi)(\alpha-l \gamma)-\psi(\delta+v \psi)(l-s) \\
& \quad+2 v(\phi-l \psi)(\beta-\gamma), \\
& I_{1}=2 v \sigma_{D}^{2}(\phi-l \psi)+4 v \sigma_{R}^{2}(\beta-\gamma)(l-s) \\
& \quad-2 \rho \sigma_{D} \sigma_{R}(v(\alpha-l \gamma)-(\delta+2 v \psi)(l-s)), \\
& J_{1}=4 v \sigma_{D}^{2} \sigma_{R}^{2}(l-s)\left(1-\rho^{2}\right), \\
& K_{1}=2 \theta(\beta-\gamma)-(\delta+v \psi)^{2}, \\
& L_{1}=2 \theta \sigma_{D}^{2}+4 v \rho \sigma_{D} \sigma_{R}(\delta+v \psi)+4 v^{2} \sigma_{R}^{2}(\beta-\gamma), \\
& F_{1}=4 v^{2} \sigma_{D}^{2} \sigma_{R}^{2}\left(1-\rho^{2}\right) .
\end{aligned}
$$

In order to have more analytical closed-form results, we assume in the rest of this paper that (5) and (9) hold which implies that $U_{1}$ and $U_{2}$ are concave functions of $p$ and $m$. Thus, we can easily derive Lemma 1.

Lemma 1. Under the scenario with full refund under consumer return, (a) the optimal pricing and modularity level decisions are given as follows:

$$
p_{1}^{*}=\frac{A_{1}+B_{1} k}{K_{1}+L_{1} k+F_{1} k^{2}}, \quad m_{1}^{*}=\frac{G_{1}+I_{1} k+J_{1} k^{2}}{K_{1}+L_{1} k+F_{1} k^{2}} .
$$

(b) The optimal pricing and modularity level decisions are linearly related to one another: $p_{1}^{*}=\xi m_{1}^{*}$, where $\xi=\left(A_{1}+\right.$ $\left.B_{1} k\right) /\left(G_{1}+I_{1} k+J_{1} k^{2}\right)$.

Lemma 1 shows the closed-form expression of the optimal decisions for the MC fashion brand when it offers full refund consumer return. Notice from Lemma $1(\mathrm{~b})$ that it is very interesting to reveal analytically that the optimal pricing decision and the optimal modularity level decisions are directly proportional to one another if $\xi>0$. Despite being complicated, we can further derive Theorem 2 by checking the respective first-order partial derivatives of the optimal decisions.

Theorem 2. Under the consumer return policy with full refund, (a) the optimal retail price is a strictly decreasing 
TABLE 2: (a) Sensitivity analysis on the effect brought by $k$ (with $\rho=-1$ ). (b) Sensitivity analysis on the effect brought by $k$ (with $\rho=-0.3$ ). (c) Sensitivity analysis on the effect brought by $k$ (with $\rho=0$ ). (d) Sensitivity analysis on the effect brought by $k$ (with $\rho=0.3$ ). (e) Sensitivity analysis on the effect brought by $k$ (with $\rho=1)$.

(a)

\begin{tabular}{lcl}
\hline$k$ & $\Delta U(\rho=-1)$ & Optimal strategy \\
\hline 0 & 3573.464 & Consumer return with full refund \\
0.0025 & 1346.646 & Consumer return with full refund \\
0.005 & 828.6819 & Consumer return with full refund \\
0.0075 & 618.2397 & Consumer return with full refund \\
0.01 & 508.4378 & Consumer return with full refund \\
0.0125 & 442.3226 & Consumer return with full refund \\
0.015 & 398.6414 & Consumer return with full refund \\
0.0175 & 367.8473 & Consumer return with full refund \\
0.02 & 345.0745 & Consumer return with full refund \\
0.0225 & 327.6048 & Consumer return with full refund \\
0.025 & 313.8096 & Consumer return with full refund \\
0.0275 & 302.6579 & Consumer return with full refund \\
0.03 & 293.4675 & Consumer return with full refund \\
0.0325 & 285.7699 & Consumer return with full refund \\
0.035 & 279.2335 & Consumer return with full refund \\
0.0375 & 273.617 & Consumer return with full refund \\
0.04 & 268.7412 & Consumer return with full refund \\
0.0425 & 264.4703 & Consumer return with full refund \\
0.045 & 260.6994 & Consumer return with full refund \\
0.0475 & 257.3464 & Consumer return with full refund \\
0.05 & 254.3461 & Consumer return with full refund \\
\hline
\end{tabular}

(b)

\begin{tabular}{lcl}
\hline$k$ & $\Delta U(\rho=-0.3)$ & Optimal strategy \\
\hline 0 & 3573.464 & Consumer return with full refund \\
0.0025 & 1077.694 & Consumer return with full refund \\
0.005 & 550.2467 & Consumer return with full refund \\
0.0075 & 351.1763 & Consumer return with full refund \\
0.01 & 253.4773 & Consumer return with full refund \\
0.0125 & 197.6467 & Consumer return with full refund \\
0.015 & 162.3939 & Consumer return with full refund \\
0.0175 & 138.5062 & Consumer return with full refund \\
0.02 & 121.4449 & Consumer return with full refund \\
0.0225 & 108.7525 & Consumer return with full refund \\
0.025 & 98.99825 & Consumer return with full refund \\
0.0275 & 91.30056 & Consumer return with full refund \\
0.03 & 85.09028 & Consumer return with full refund \\
0.0325 & 79.98568 & Consumer return with full refund \\
0.035 & 75.72236 & Consumer return with full refund \\
0.0375 & 72.11208 & Consumer return with full refund \\
0.04 & 69.01759 & Consumer return with full refund \\
0.0425 & 66.33674 & Consumer return with full refund \\
0.045 & 63.99209 & Consumer return with full refund \\
0.0475 & 61.924 & Consumer return with full refund \\
0.05 & 60.08586 & Consumer return with full refund \\
\hline
\end{tabular}

(c)

\begin{tabular}{lcc}
\hline$k$ & $\Delta U(\rho=0)$ & Optimal strategy \\
\hline 0 & 3573.464 & Consumer return with full refund \\
0.0025 & 972.1282 & Consumer return with full refund \\
\hline
\end{tabular}

(c) Continued.

\begin{tabular}{lcc}
\hline$k$ & $\Delta U(\rho=0)$ & Optimal strategy \\
\hline 0.005 & 447.7589 & Consumer return with full refund \\
0.0075 & 257.8076 & Consumer return with full refund \\
0.01 & 168.0751 & Consumer return with full refund \\
0.0125 & 118.62 & Consumer return with full refund \\
0.015 & 88.45607 & Consumer return with full refund \\
0.0175 & 68.6862 & Consumer return with full refund \\
0.02 & 55.01266 & Consumer return with full refund \\
0.0225 & 45.15184 & Consumer return with full refund \\
0.025 & 37.79863 & Consumer return with full refund \\
0.0275 & 32.16292 & Consumer return with full refund \\
0.03 & 27.74351 & Consumer return with full refund \\
0.0325 & 24.2099 & Consumer return with full refund \\
0.035 & 21.33693 & Consumer return with full refund \\
0.0375 & 18.96689 & Consumer return with full refund \\
0.04 & 16.98659 & Consumer return with full refund \\
0.0425 & 15.31309 & Consumer return with full refund \\
0.045 & 13.88447 & Consumer return with full refund \\
0.0475 & 12.65375 & Consumer return with full refund \\
0.05 & 11.58473 & Consumer return with full refund \\
\hline
\end{tabular}

(d)

\begin{tabular}{lcl}
\hline$k$ & $\Delta U(\rho=0.3)$ & Optimal strategy \\
\hline 0 & 3573.464 & Consumer return with full refund \\
0.0025 & 872.1531 & Consumer return with full refund \\
0.005 & 354.8006 & Consumer return with full refund \\
0.0075 & 176.2229 & Consumer return with full refund \\
0.01 & 95.8788 & Consumer return with full refund \\
0.0125 & 53.7666 & Consumer return with full refund \\
0.015 & 29.38607 & Consumer return with full refund \\
0.0175 & 14.25304 & Consumer return with full refund \\
0.02 & 4.366616 & Consumer return with full refund \\
0.0225 & -2.34816 & No return \\
0.025 & -7.0484 & No return \\
0.0275 & -10.4174 & No return \\
0.03 & -12.8778 & No return \\
0.0325 & -14.7011 & No return \\
0.035 & -16.0677 & No return \\
0.0375 & -17.1003 & No return \\
0.04 & -17.8848 & No return \\
0.0425 & -18.4824 & No return \\
0.045 & -18.9374 & No return \\
0.0475 & -19.2824 & No return \\
0.05 & -19.542 & No return \\
\hline & &
\end{tabular}

(e)

\begin{tabular}{lcl}
\hline$k$ & $\Delta U(\rho=1)$ & Optimal strategy \\
\hline 0 & 3573.464 & Consumer return with full refund \\
0.0005 & 2374.058 & Consumer return with full refund \\
0.001 & 1653.531 & Consumer return with full refund \\
0.0015 & 1190.932 & Consumer return with full refund \\
0.002 & 878.7702 & Consumer return with full refund \\
0.0025 & 659.8301 & Consumer return with full refund \\
0.003 & 501.4762 & Consumer return with full refund \\
0.0035 & 384.0431 & Consumer return with full refund \\
0.004 & 295.1391 & Consumer return with full refund \\
0.0045 & 226.6607 & Consumer return with full refund \\
0.005 & 173.1403 & Consumer return with full refund \\
\hline
\end{tabular}


(e) Continued.

\begin{tabular}{lcl}
\hline$k$ & $\Delta U(\rho=1)$ & Optimal strategy \\
\hline 0.0055 & 130.7888 & Consumer return with full refund \\
0.006 & 96.91849 & Consumer return with full refund \\
0.0065 & 69.58424 & Consumer return with full refund \\
0.007 & 47.35295 & Consumer return with full refund \\
0.0075 & 29.15195 & Consumer return with full refund \\
0.008 & 14.16698 & Consumer return with full refund \\
0.0085 & 1.772005 & Consumer return with full refund \\
0.009 & -8.51977 & No return \\
0.0095 & -17.0909 & No return \\
0.01 & -24.2446 & No return \\
0.0105 & -30.2239 & No return \\
0.011 & -35.2243 & No return \\
0.0115 & -39.4048 & No return \\
0.012 & -42.8955 & No return \\
0.0125 & -45.8034 & No return \\
0.013 & -48.217 & No return \\
0.0135 & -50.2098 & No return \\
0.014 & -51.8436 & No return \\
0.0145 & -53.17 & No return \\
0.015 & -54.2326 & No return \\
\hline
\end{tabular}

(increasing) function of the service charge lif $-\gamma \theta-v \delta \psi+$ $2 k v \sigma_{R}^{2}(\delta-v \gamma)+\left(2 k \rho \sigma_{D} \sigma_{R}-\psi\right)\left(\theta+v^{2} \psi\right)<0$ (sufficient conditions are $\delta-v \gamma<0$ and $\left.2 k \rho \sigma_{D} \sigma_{R}-\psi<0\right)$. (b) The optimal retail price is a strictly increasing (increasing) function of the salvage values if $\theta>2 k v \delta \sigma_{R}^{2} /\left(\psi-2 k \rho \sigma_{D} \sigma_{R}\right)$.

In parallel to the scenario with consumer return, we can derive Lemma 3 by the same logic.

Lemma 3. Under the no consumer return scenario, (a) the optimal pricing and modularity level decisions are given as follows $p_{2}^{*}=\alpha \theta /\left(2 \theta\left(\beta+k \sigma_{D}^{2}\right)-\delta^{2}\right)$ and $m_{2}^{*}=\alpha \delta /(2 \theta(\beta+$ $\left.\left.k \sigma_{D}^{2}\right)-\delta^{2}\right)$. (b) The relationship between the optimal pricing and the optimal modularity level is given by $p_{2}^{*}=(\theta / \delta) m_{2}^{*}$.

Lemma 3 shows the closed-form expression of the optimal decisions for the MC fashion brand when there is no consumer return. Similar to the case with consumer return, it is very interesting to note that the resulting optimal retail price is directly proportional to the optimal modularity level. In other words, Lemmas 1(b) and 3(b) both reveal that it is optimal for the MC fashion brand to charge a higher price if more options are available to consumers (as quantified by the level of modularity in the model) no matter whether the "consumer return with full refund" (p.s.: for the case with consumer return and full refund, this result holds whenever $\xi$ is positive) or "no return" strategy is imposed. This supplements the common belief that fashion brands can charge a premium by offering MC (compared to mass production) because of having more variety of options for consumers. With the result in Lemma 3, we can further derive Theorem 4.

Theorem 4. Under the no consumer return scenario, (a) If $k$ increases, both $p_{2}^{*}$ and $m_{2}^{*}$ will decrease. (b) If $\beta$ or $\sigma_{D}$ increases,
TABLE 3: (a) Sensitivity analysis on the effect brought by $\sigma_{R}$ (with $\rho=-1$ ). (b) Sensitivity analysis on the effect brought by $\sigma_{R}$ (with $\rho=-0.3$ ). (c) Sensitivity analysis on the effect brought by $\sigma_{R}$ (with $\rho=0$ ). (d) Sensitivity analysis on the effect brought by $\sigma_{R}$ (with $\rho=0.3$ ). (e) Sensitivity analysis on the effect brought by $\sigma_{R}$ (with $\rho=1)$.

(a)

\begin{tabular}{lcc}
\hline$\sigma_{R}$ & $\Delta U(\rho=-1)$ & Optimal strategy \\
\hline 0 & 57.47614 & Consumer return with full refund \\
5 & 112.6601 & Consumer return with full refund \\
10 & 178.8924 & Consumer return with full refund \\
15 & 256.321 & Consumer return with full refund \\
20 & 345.0745 & Consumer return with full refund \\
25 & 445.2616 & Consumer return with full refund \\
30 & 556.9698 & Consumer return with full refund \\
35 & 680.2648 & Consumer return with full refund \\
40 & 815.1897 & Consumer return with full refund \\
45 & 961.7641 & Consumer return with full refund \\
50 & 1119.983 & Consumer return with full refund \\
55 & 1289.818 & Consumer return with full refund \\
60 & 1471.215 & Consumer return with full refund \\
65 & 1664.095 & Consumer return with full refund \\
70 & 1868.354 & Consumer return with full refund \\
75 & 2083.861 & Consumer return with full refund \\
80 & 2310.462 & Consumer return with full refund \\
85 & 2547.977 & Consumer return with full refund \\
90 & 2796.201 & Consumer return with full refund \\
95 & 3054.907 & Consumer return with full refund \\
100 & 3323.843 & Consumer return with full refund \\
\hline
\end{tabular}

(b)

\begin{tabular}{lcl}
\hline$\sigma_{R}$ & $\Delta U(\rho=-0.3)$ & Optimal strategy \\
\hline 0 & 57.47614 & Consumer return with full refund \\
5 & 72.71861 & Consumer return with full refund \\
10 & 88.54183 & Consumer return with full refund \\
15 & 104.8257 & Consumer return with full refund \\
20 & 121.4449 & Consumer return with full refund \\
25 & 138.2716 & Consumer return with full refund \\
30 & 155.1791 & Consumer return with full refund \\
35 & 172.0444 & Consumer return with full refund \\
40 & 188.7511 & Consumer return with full refund \\
45 & 205.1917 & Consumer return with full refund \\
50 & 221.269 & Consumer return with full refund \\
55 & 236.8976 & Consumer return with full refund \\
60 & 252.0048 & Consumer return with full refund \\
65 & 266.5306 & Consumer return with full refund \\
70 & 280.4274 & Consumer return with full refund \\
75 & 293.6601 & Consumer return with full refund \\
80 & 306.2044 & Consumer return with full refund \\
85 & 318.0468 & Consumer return with full refund \\
90 & 329.1825 & Consumer return with full refund \\
95 & 339.615 & Consumer return with full refund \\
100 & 349.3545 & Consumer return with full refund \\
\hline
\end{tabular}

(c)

\begin{tabular}{lcc}
\hline$\sigma_{R}$ & $\Delta U(\rho=0)$ & Optimal strategy \\
\hline 0 & 57.47614 & Consumer return with full refund \\
5 & 57.31906 & Consumer return with full refund \\
\hline
\end{tabular}


(c) Continued.

\begin{tabular}{lcc}
\hline$\sigma_{R}$ & $\Delta U(\rho=0)$ & Optimal strategy \\
\hline 10 & 56.85037 & Consumer return with full refund \\
15 & 56.07753 & Consumer return with full refund \\
20 & 55.01266 & Consumer return with full refund \\
25 & 53.67209 & Consumer return with full refund \\
30 & 52.0757 & Consumer return with full refund \\
35 & 50.24625 & Consumer return with full refund \\
40 & 48.20862 & Consumer return with full refund \\
45 & 45.98904 & Consumer return with full refund \\
50 & 43.61431 & Consumer return with full refund \\
55 & 41.11118 & Consumer return with full refund \\
60 & 38.5057 & Consumer return with full refund \\
65 & 35.82278 & Consumer return with full refund \\
70 & 33.08575 & Consumer return with full refund \\
75 & 30.31611 & Consumer return with full refund \\
80 & 27.53335 & Consumer return with full refund \\
85 & 24.75483 & Consumer return with full refund \\
90 & 21.99579 & Consumer return with full refund \\
95 & 19.26937 & Consumer return with full refund \\
100 & 16.58673 & Consumer return with full refund \\
\hline
\end{tabular}

(d)

\begin{tabular}{lcl}
\hline$\sigma_{R}$ & $\Delta U(\rho=0.3)$ & Optimal strategy \\
\hline 0 & 57.47614 & Consumer return with full refund \\
1 & 54.50733 & Consumer return with full refund \\
2 & 51.56711 & Consumer return with full refund \\
3 & 48.65629 & Consumer return with full refund \\
4 & 45.77567 & Consumer return with full refund \\
5 & 42.92605 & Consumer return with full refund \\
6 & 40.10819 & Consumer return with full refund \\
7 & 37.32283 & Consumer return with full refund \\
8 & 34.57069 & Consumer return with full refund \\
9 & 31.85248 & Consumer return with full refund \\
10 & 29.16887 & Consumer return with full refund \\
11 & 26.52051 & Consumer return with full refund \\
12 & 23.90802 & Consumer return with full refund \\
13 & 21.33201 & Consumer return with full refund \\
14 & 18.79306 & Consumer return with full refund \\
15 & 16.29172 & Consumer return with full refund \\
\hline
\end{tabular}

(e)

\begin{tabular}{lcl}
\hline$\sigma_{R}$ & $\Delta U(\rho=1)$ & Optimal strategy \\
\hline 0 & 57.47614 & Consumer return with full refund \\
1 & 47.75089 & Consumer return with full refund \\
2 & 38.4596 & Consumer return with full refund \\
3 & 29.60083 & Consumer return with full refund \\
4 & 21.17313 & Consumer return with full refund \\
5 & 13.175 & Consumer return with full refund \\
6 & 5.604925 & Consumer return with full refund \\
7 & -1.53862 & No return \\
8 & -8.25721 & No return \\
9 & -14.5524 & No return \\
10 & -20.4259 & No return \\
11 & -25.8792 & No return \\
12 & -30.914 & No return \\
13 & -35.532 & No return \\
14 & -39.735 & No return \\
15 & -43.5246 & No return \\
\hline
\end{tabular}

TABLE 4: (a) Sensitivity analysis on the effect brought by $\sigma_{D}$ (with $\rho=-1$ ). (b) Sensitivity analysis on the effect brought by $\sigma_{D}$ (with $\rho=-0.3$ ). (c) Sensitivity analysis on the effect brought by $\sigma_{D}$ (with $\rho=0$ ). (d) Sensitivity analysis on the effect brought by $\sigma_{D}$ (with $\rho=0.3$ ). (e) Sensitivity analysis on the effect brought by $\sigma_{D}$ (with $\rho=1)$.

(a)

\begin{tabular}{lcl}
\hline$\sigma_{D}$ & $\Delta U(\rho=-1)$ & Optimal strategy \\
\hline 20 & 3449.186 & Consumer return with full refund \\
30 & 2594.183 & Consumer return with full refund \\
40 & 1844.719 & Consumer return with full refund \\
50 & 1307.854 & Consumer return with full refund \\
60 & 946.619 & Consumer return with full refund \\
70 & 705.1343 & Consumer return with full refund \\
80 & 541.0528 & Consumer return with full refund \\
90 & 426.7885 & Consumer return with full refund \\
100 & 345.0745 & Consumer return with full refund \\
110 & 285.1112 & Consumer return with full refund \\
120 & 240.0424 & Consumer return with full refund \\
130 & 205.4228 & Consumer return with full refund \\
140 & 178.3037 & Consumer return with full refund \\
150 & 156.6838 & Consumer return with full refund \\
160 & 139.1748 & Consumer return with full refund \\
170 & 124.7939 & Consumer return with full refund \\
180 & 112.8319 & Consumer return with full refund \\
190 & 102.7681 & Consumer return with full refund \\
200 & 94.2139 & Consumer return with full refund \\
\hline
\end{tabular}

(b)

\begin{tabular}{lcc}
\hline$\sigma_{D}$ & $\Delta U(\rho=-0.3)$ & Optimal strategy \\
\hline 20 & 2450.895 & Consumer return with full refund \\
30 & 1608.23 & Consumer return with full refund \\
40 & 1016.281 & Consumer return with full refund \\
50 & 650.045 & Consumer return with full refund \\
60 & 430.0031 & Consumer return with full refund \\
70 & 296.1204 & Consumer return with full refund \\
80 & 212.2569 & Consumer return with full refund \\
90 & 157.9012 & Consumer return with full refund \\
100 & 121.4449 & Consumer return with full refund \\
110 & 96.19273 & Consumer return with full refund \\
120 & 78.1769 & Consumer return with full refund \\
130 & 64.97559 & Consumer return with full refund \\
140 & 55.06682 & Consumer return with full refund \\
150 & 47.46713 & Consumer return with full refund \\
160 & 41.52439 & Consumer return with full refund \\
170 & 36.7957 & Consumer return with full refund \\
180 & 32.9736 & Consumer return with full refund \\
190 & 29.84028 & Consumer return with full refund \\
200 & 27.23856 & Consumer return with full refund \\
\hline
\end{tabular}

(c)

\begin{tabular}{ccl}
\hline$\sigma_{D}$ & $\Delta U(\rho=0)$ & Optimal strategy \\
\hline 20 & 2084.977 & Consumer return with full refund \\
30 & 1266.59 & Consumer return with full refund \\
40 & 741.2984 & Consumer return with full refund \\
50 & 438.7885 & Consumer return with full refund \\
60 & 268.2584 & Consumer return with full refund \\
70 & 170.537 & Consumer return with full refund \\
\hline
\end{tabular}


(c) Continued.

\begin{tabular}{lcl}
\hline$\sigma_{D}$ & $\Delta U(\rho=0)$ & Optimal strategy \\
\hline 80 & 112.777 & Consumer return with full refund \\
90 & 77.41918 & Consumer return with full refund \\
100 & 55.01266 & Consumer return with full refund \\
110 & 40.34647 & Consumer return with full refund \\
120 & 30.4589 & Consumer return with full refund \\
130 & 23.61273 & Consumer return with full refund \\
140 & 18.75729 & Consumer return with full refund \\
150 & 15.23858 & Consumer return with full refund \\
160 & 12.63853 & Consumer return with full refund \\
170 & 10.68328 & Consumer return with full refund \\
180 & 9.189367 & Consumer return with full refund \\
190 & 8.031324 & Consumer return with full refund \\
200 & 7.121739 & Consumer return with full refund \\
\hline
\end{tabular}

(d)

\begin{tabular}{lcl}
\hline$\sigma_{D}$ & $\Delta U(\rho=0.3)$ & Optimal strategy \\
\hline 20 & 1752.936 & Consumer return with full refund \\
30 & 968.2728 & Consumer return with full refund \\
40 & 508.8139 & Consumer return with full refund \\
50 & 264.978 & Consumer return with full refund \\
60 & 138.1811 & Consumer return with full refund \\
70 & 71.41194 & Consumer return with full refund \\
80 & 35.41802 & Consumer return with full refund \\
90 & 15.54633 & Consumer return with full refund \\
100 & 4.366616 & Consumer return with full refund \\
110 & -1.98807 & No return \\
120 & -5.59179 & No return \\
130 & -7.59094 & No return \\
140 & -8.63718 & No return \\
150 & -9.1104 & No return \\
160 & -9.237 & No return \\
170 & -9.15444 & No return \\
180 & -8.9475 & No return \\
190 & -8.66903 & No return \\
200 & -8.35226 & No return \\
\hline
\end{tabular}

(e)

\begin{tabular}{lcl}
\hline$\sigma_{D}$ & $\Delta U(\rho=1)$ & Optimal strategy \\
\hline 20 & 1099.709 & Consumer return with full refund \\
25 & 704.3688 & Consumer return with full refund \\
30 & 425.6345 & Consumer return with full refund \\
35 & 238.2991 & Consumer return with full refund \\
40 & 116.6536 & Consumer return with full refund \\
45 & 39.76602 & Consumer return with full refund \\
50 & -7.6261 & No return \\
55 & -35.9901 & No return \\
60 & -52.2552 & No return \\
65 & -60.9155 & No return \\
70 & -64.8518 & No return \\
\hline
\end{tabular}

both $p_{2}^{*}$ and $m_{2}^{*}$ will decrease. (c) If $\alpha$ increases, both $p_{2}^{*}$ and $m_{2}^{*}$ will increase. (d) If $\theta$ increases, $m_{2}^{*}$ will decrease. (e) If $\delta$ increases, $p_{2}^{*}$ will decrease.

From Theorem 2, we can see that, for the scenario when the MC fashion brand offers consumer return, the optimal decisions on retail price and modularity level depend highly on the salvage value and the return service charge. From
Theorem 4, we can observe that for the scenario when the MC fashion brand does not offer consumer return, the optimal decisions on retail price and modularity level depend highly on different market parameters such as the primary demand, the price-demand sensitivity coefficient, the modularitydemand sensitivity coefficient, and the modularity cost sensitivity coefficient; the corresponding relationships are shown in closed-form as summarized by Theorem 4 .

After we have analyzed the separate scenarios, we now examine a critical research question of this paper on whether the MC fashion brand should offer consumer return with full refund or no return. To address this question, we employ the analytical results above and define $\Delta U \equiv U_{1}\left(p_{1}^{*}, m_{1}^{*}\right)-$ $U_{2}\left(p_{2}^{*}, m_{2}^{*}\right)$. Obviously, whenever $\Delta U$ is strictly positive, we have $U_{1}\left(p_{1}^{*}, m_{1}^{*}\right)>U_{2}\left(p_{2}^{*}, m_{2}^{*}\right)$ which means that it is better to implement the consumer return policy with full refund, compared to the strategy of offering no return. As such, $\Delta U$ represents the amount of benefit that can be brought by employing consumer return with full refund (as compared to the no return no refund scenario) in MV domain.

\section{Numerical Sensitivity Analysis}

Owing to the complexity of the analytical expression of $\Delta U$, we have to employ extensive numerical analysis to reveal under what conditions the strategy of "implementing consumer return with full refund" will outperform the strategy of "no return and no refund." In determining the set of numerical values in conducting the analysis, we make reference to the data set as employed in $[28,32]$. To be specific, we set the parameters as in Table 1.

We focus on analyzing how the degree of risk aversion $k$, the return uncertainty $\sigma_{R}$, and the demand uncertainty $\sigma_{D}$ affect the value of $\Delta U$ (which in turn determines the optimal strategy) because they are the most critical parameters related to the consumer return policy under MC. We also explore the problem with "positive, zero, and negative $\rho$ " because a different sign of $\rho$ can significantly affect the finding. The results are shown in Tables 2 to 4 and also plotted in Figures 1 to 3 , respectively. Notice that the numerical range of each parameter is set with reference to the conditions with which the mean-variance objective function remains concave and the optimal pricing and modularity level decisions are all feasible.

From Tables 2(a) to 2(e), it is interesting to note that the no return scenario would outperform the consumer return scenario with full refund only when $\rho$ and $k$ are both big enough. In other words, when $\rho \leq 0$, consumer return with full refund is more preferred to the MC fashion brand irrespective of the degree of risk aversion. Only under the case when $\rho \geq 0.3$, for a sufficiently risk averse MC fashion brand (i.e. with a sufficiently big $k$ ), having no return is better than the case with consumer return with full refund. This result is partially intuitive as it indicates that a sufficiently risk averse MC fashion brand will prefer no return to having consumer return because the corresponding risk from consumer return is too high to bear. However, our result also indicates the important role played by the "very strong mediating factor" 


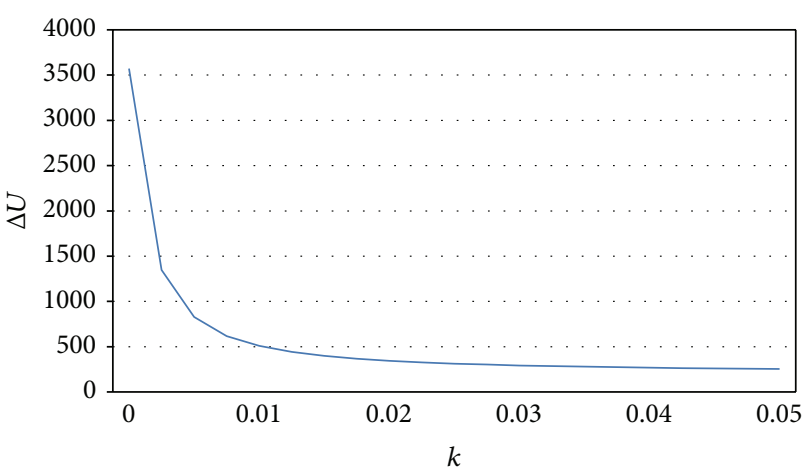

(a)

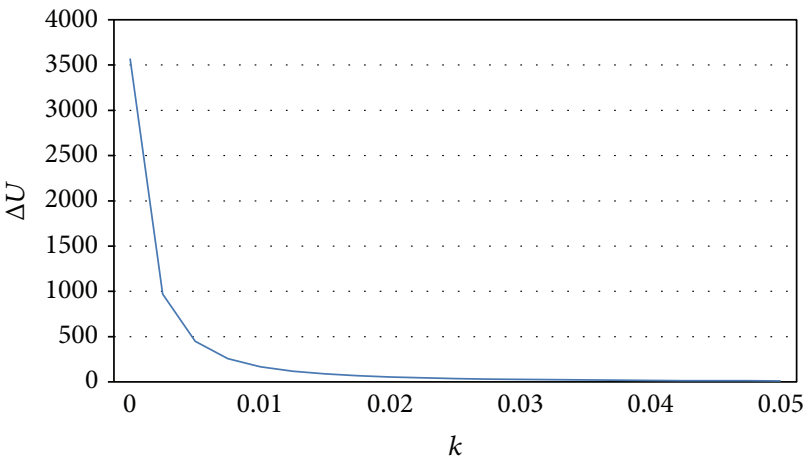

(c)

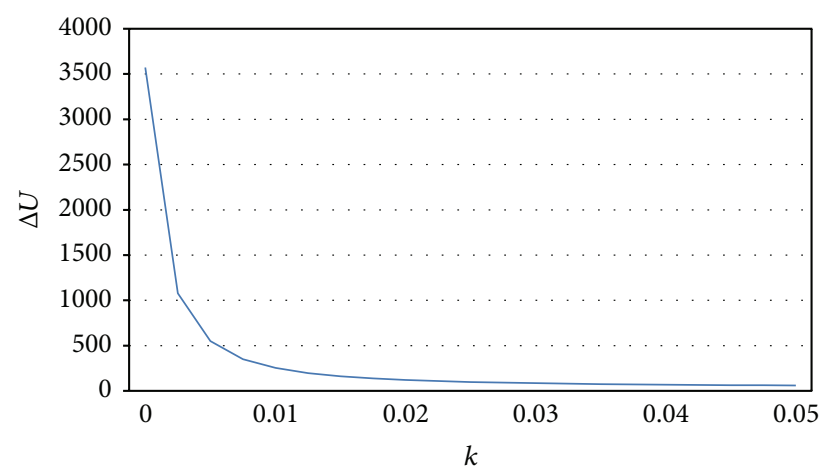

(b)

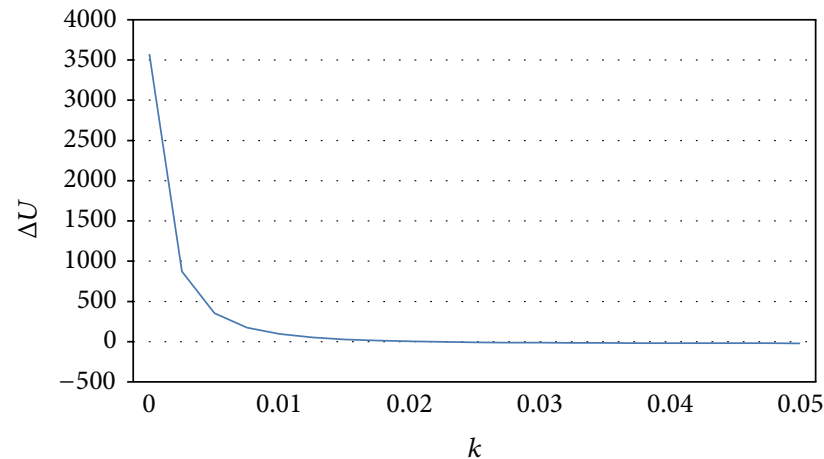

(d)

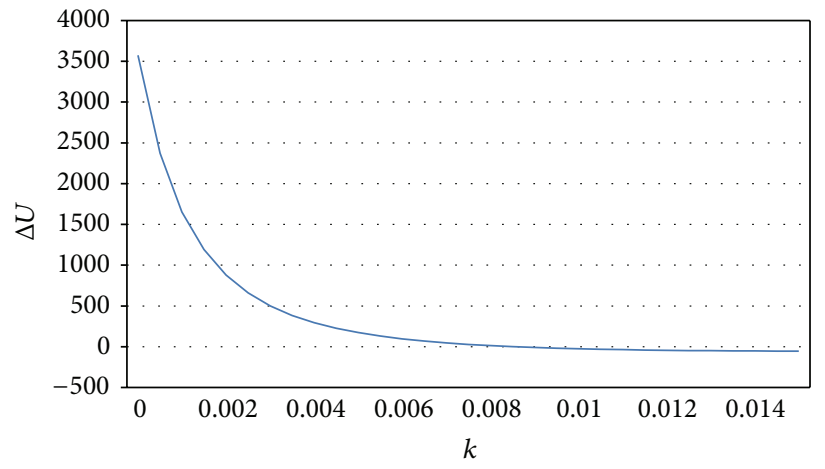

(e)

FIGURE 1: (a) Sensitivity analysis on the effect brought by $k$ (with $\rho=-1$ ). (b) Sensitivity analysis on the effect brought by $k$ (with $\rho=-0.3$ ). (c) Sensitivity analysis on the effect brought by $k$ (with $\rho=0$ ). (d) Sensitivity analysis on the effect brought by $k$ (with $\rho=0.3$ ). (e) Sensitivity analysis on the effect brought by $k$ (with $\rho=1$ ).

$\rho$ which can affect the result very significantly. In addition, as observed from Figures 1(a) to 1(e), for all cases, when $k$ increases, $\Delta U$ decreases which implies that the amount of benefit that can be brought by employing consumer return with full refund (as compared to the no return no refund scenario) in MV domain is decreasing with the MC fashion brand's degree of risk aversion.

From Tables 3(a) to 3(e), we can observe that when $\rho$ is small (i.e., $\rho \leq 0.3$ ), the consumer return scenario with full refund is the preferred choice for all values of $\sigma_{R}$ under study. When $\rho=1$, (i) we observe that the consumer return scenario with full refund is still the preferred choice when $\sigma_{R}$ is small. (ii) If $\sigma_{R}$ is sufficiently high, then the no return scenario outperforms the full refund scenario. Again, similar to our prior findings, $\rho$ is a very important "mediating factor" which can substantially affect the optimal choice of whether to adopt no return or return with full refund when the return uncertainty varies. From Figures 2(a) to 2(e), we can observe that, (i) for $\rho<0$, when $\sigma_{R}$ increases, $\Delta U$ increases which means that the respective amount of benefit that can be brought by employing consumer return with full refund (as compared to the no return no refund scenario) in MV domain is increasing with the return uncertainty. (ii) On the contrary, for $\rho \geq 0$, when $\sigma_{R}$ increases, $\Delta U$ decreases which indicates that the effect as brought by increasing $\sigma_{R}$ on $\Delta U$ is just reverted when $\rho \geq 0$ (compared to the case when $\rho<0$ ). 


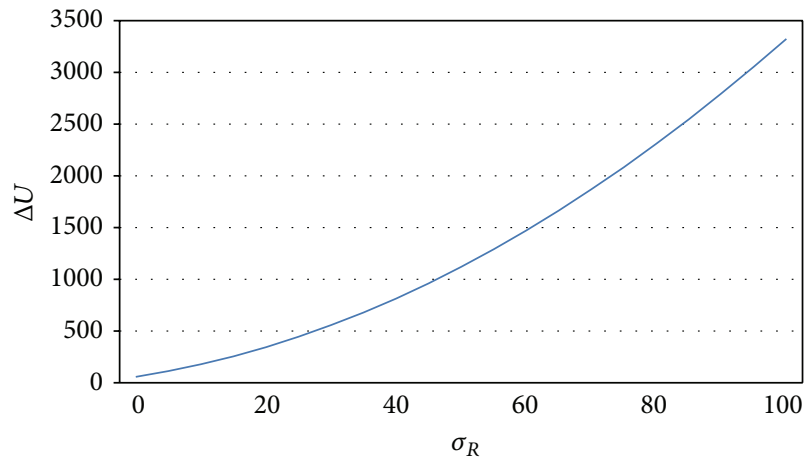

(a)

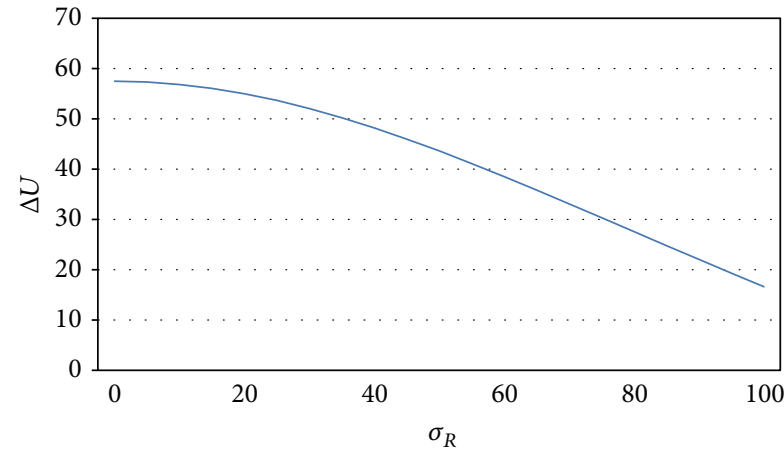

(c)

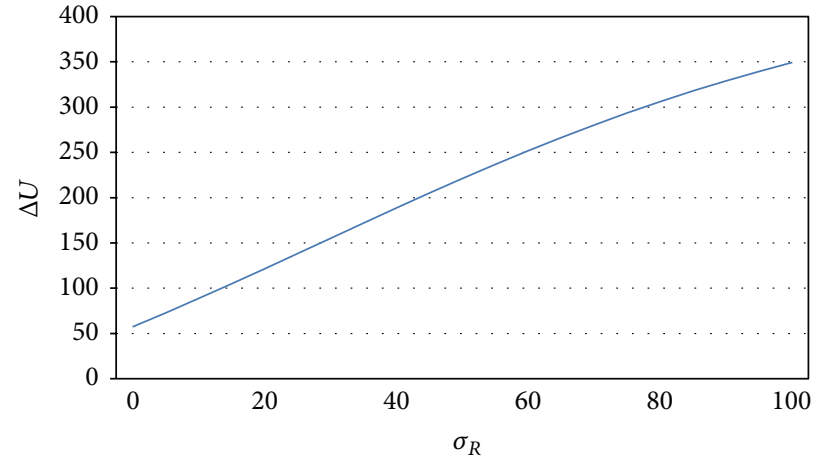

(b)

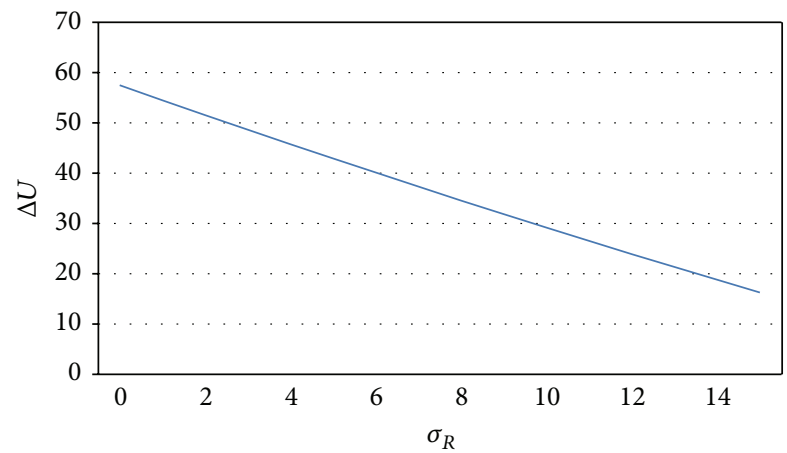

(d)

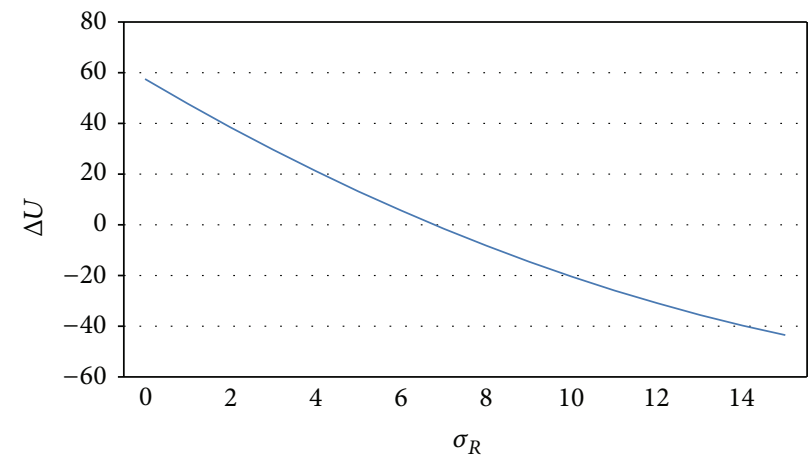

(e)

FIGURE 2: (a) Sensitivity analysis on the effect brought by $\sigma_{R}$ (with $\rho=-1$ ). (b) Sensitivity analysis on the effect brought by $\sigma_{R}$ (with $\rho=-0.3$ ). (c) Sensitivity analysis on the effect brought by $\sigma_{R}$ (with $\rho=0$ ). (d) Sensitivity analysis on the effect brought by $\sigma_{R}$ (with $\rho=0.3$ ). (e) Sensitivity analysis on the effect brought by $\sigma_{R}$ (with $\rho=1$ ).

From Tables 4(a) to 4(e), it is interesting to note that the impact on the optimal choice of return policy (no return versus return with full refund) as brought by the demand uncertainty is rather similar to the return uncertainty. The consumer return with full refund scenario is the preferred choice for all demand uncertainty $\left(\sigma_{D}\right)$ values under study when $\rho \leq$ 0 . When $\rho \geq 0.3$, we find that, (i) the consumer return scenario with full refund is still the preferred choice when $\sigma_{D}$ is small. (ii) If $\sigma_{D}$ is sufficiently big, the no return scenario outperforms the full refund scenario. As observed from Figures 3(a) to 3(e), we notice that different from the case with $\sigma_{R}, \Delta U$ is always a decreasing function of $\sigma_{D}$ irrespective of whether $\rho$ is positive or negative. In other words, the amount of benefit that can be brought by employing consumer return with full refund (as compared to the no return no refund scenario) in MV domain is decreasing with the demand uncertainty for all $\rho$.

\section{Conclusion}

In this paper, we have explored the stochastic fashion MC service programme with the consideration of consumer demand uncertainty and risk aversion of the company. We have obtained a number of important findings and insights. For example, for the scenario with full refund and return, we have derived the closed-form analytical optimal decisions in retail pricing and modularity level. We have also 


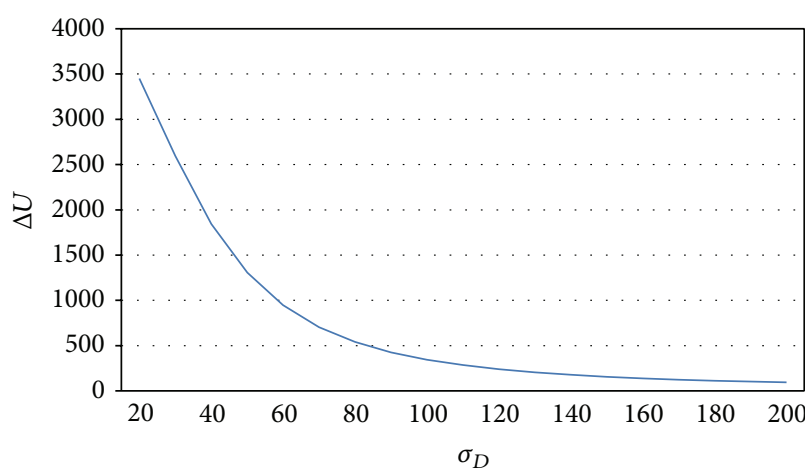

(a)

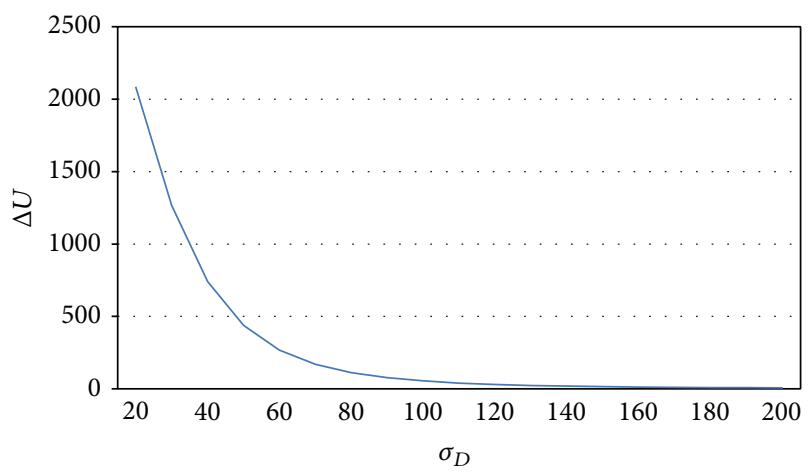

(c)

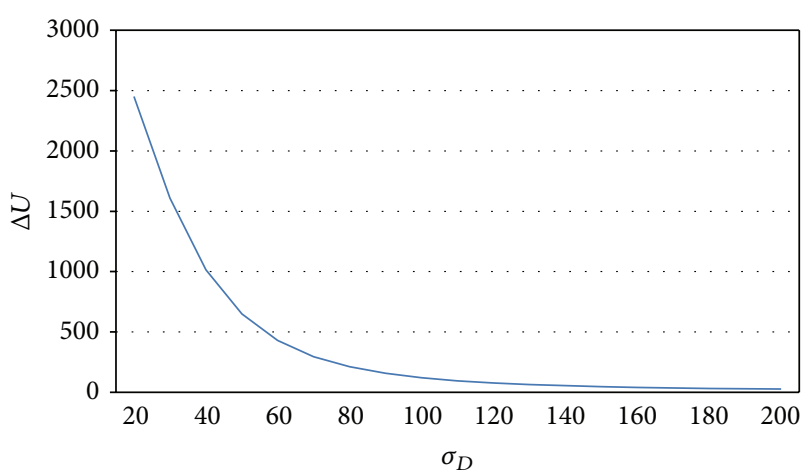

(b)

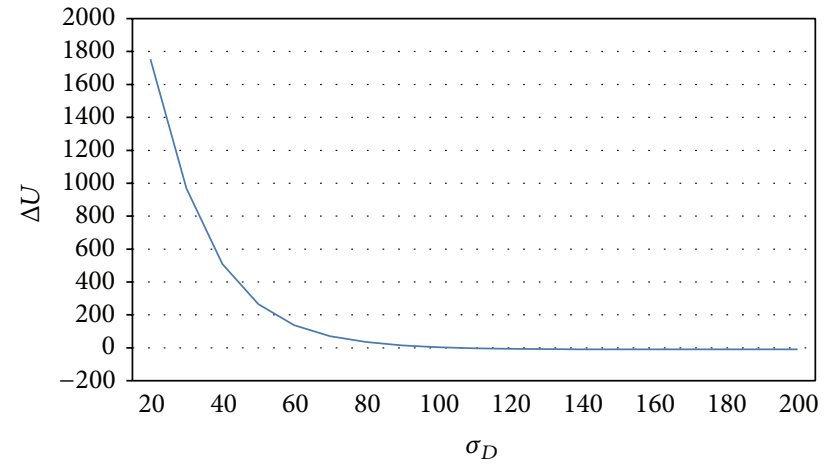

(d)

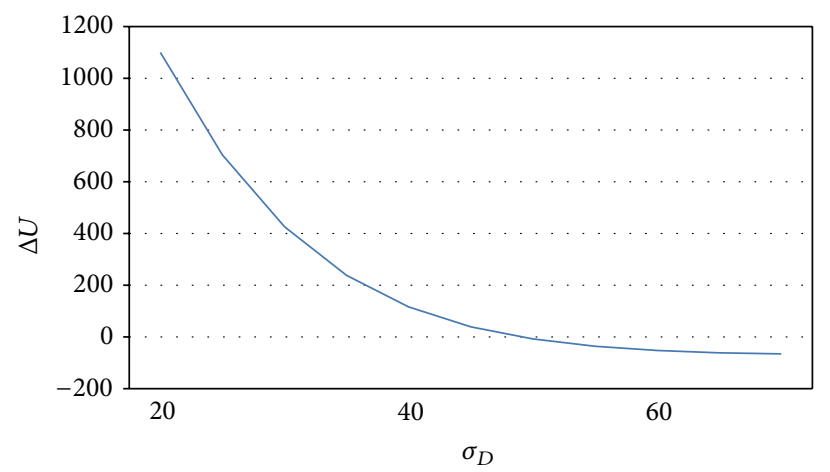

(e)

FIGURE 3: (a) Sensitivity analysis on the effect brought by $\sigma_{D}$ (with $\rho=-1$ ). (b) Sensitivity analysis on the effect brought by $\sigma_{D}$ (with $\rho=-0.3$ ). (c) Sensitivity analysis on the effect brought by $\sigma_{D}$ (with $\rho=0$ ). (d) Sensitivity analysis on the effect brought by $\sigma_{D}$ (with $\rho=0.3$ ). (e) Sensitivity analysis on the effect brought by $\sigma_{D}$ (with $\left.\rho=1\right)$.

revealed the analytical conditions under which the optimal retail price and the optimal modularity level would vary monotonically with respect to the return service charge and the salvage value. For the scenario when there is no refund and no return, we have obtained the neat closed-form expressions of the optimal pricing and modularity decisions. Our analysis has also indicated that the optimal retail price and the optimal modularity level are decreasing in the MC fashion brand's degree of risk aversion, the demand uncertainty, and the price-sensitivity coefficient. In addition, for both scenarios, we have interestingly found that the optimal retail pricing decision is linearly proportional to the optimal modularity decision. Finally, we have discovered that whether the risk averse MC fashion brand would prefer offering consumer return with full refund to no return depends critically on the demand-return correlation parameter $\rho$.

For future research, we believe that it can be done in a number of ways. First, it will be interesting to examine the MC schemes with multiple players under competition in a game-theoretic setting. Second, it will be promising to explore empirically more operational and marketing factors which may affect the demand of MC fashion products. After that, new analytical models based on the new empirical results can be constructed for further in-depth theoretical analysis. 


\section{Notation}

$p:$ The unit retail price

$m$ : The level of modularity

$\widetilde{D}_{1}$ : The market demand in the presence of consumer return with full refund

$\widetilde{D}_{2}$ : The market demand in the absence of consumer return

l: $\quad$ The service charge per return

$\alpha$ : The primary demand which depends on factors such as product quality, brand image, and the general market factors, $\alpha>0$

$\beta$ : The price-demand sensitivity coefficient, $\beta>0$

$\gamma$ : The (consumer return) refund-rate-demand sensitivity coefficient, $\beta>\gamma>0$

$\delta$ : The modularity-demand sensitivity coefficient, $\delta>0$

$\varepsilon_{D}$ : A bounded continuous random variable which represents the demand uncertainty with zero mean and variance $\sigma_{D}^{2}$

$\phi$ : The base return number which is independent of the refund rate, $\phi>0$

$\psi$ : The return number sensitivity coefficient, $\psi>0$

$\widetilde{R}: \quad$ The return quantity

$\varepsilon_{R}$ : A bounded continuous random variable which represents the uncertainty regarding the number of return with zero mean and variance $\sigma_{R}^{2}$

$\theta$ : The modularity-cost sensitivity coefficient, $\theta>0$

$v$ : The unit modularity level $m$ dependent reusability value of the returned product which depends on $v>0$

$s$ : The unit reusability value of the returned product which is independent of $m, s>0$

$S(\cdot)$ : The salvage value function

$C(\cdot)$ : The modularity cost function.

\section{Conflict of Interests}

In this paper, names of some real world companies are mentioned. The coauthors declare that there is no conflict of interests in citing these companies. The coauthors need to cite them because they are well-known examples to illustrate the real-world relevance of the analysis.

\section{Acknowledgments}

S. Ren's research is supported by the funding provided by The Hong Kong Polytechnic University. T.-M. Choi's research is partially supported by the research funding provided by RGC (HK), under grant no. PolyU 5420/10H. The authors are grateful for the constructive comments made by the guest editor and the three anonymous reviewers.

\section{References}

[1] B. Dong, H. Jia, Z. Li, and K. C. Dong, "Implementing mass customization in garment industry," Systems Engineering Procedia, vol. 3, pp. 372-380, 2012.

[2] A. Alptekinoğlu and C. J. Corbett, "Mass customization vs. mass production: variety and price competition," Manufacturing and Service Operations Management, vol. 10, no. 2, pp. 204-217, 2008.

[3] R. Duray, P. T. Ward, G. W. Miligan, and W. L. Berry, "Approaches to mass customization: configurations and empirical validation," Journal of Operations Management, vol. 18, no. 6, pp. 605625, 2000.

[4] J. Jiao, Q. Ma, and M. M. Tseng, “Towards high-value added products and services: mass customization and beyond," Technovation, vol. 23, no. 10, pp. 809-821, 2003.

[5] B. J. Pine, B. Victor, and A. C. Boynton, "Making mass customization work," Harvard Business Review, vol. 71, no. 5, pp. 108-118, 1993.

[6] R. S. Selladurai, "Mass customization in operations management: oxymoron or reality?” Omega, vol. 32, no. 4, pp. 295-300, 2004.

[7] K. Liao, X. Deng, and E. Marsillac, "Factors thatinfluence Chinese automotive suppliers'mass customization capabilities," International Journal of Production Economics. In press.

[8] H. T. Yeung, T. M. Choi, and C. H. Chiu, "Innovative mass customization in fashion," in Innovative Quick Response Programs in Logistics and Supply Chain Management, T. C. E. Cheng and T. M. Choi, Eds., pp. 423-454, Springer, 2010.

[9] T. M. Choi and S. Sethi, "Innovative quick response programs: a review," International Journal of Production Economics, vol. 127, no. 1, pp. 1-12, 2010.

[10] T. M. Choi, "Quick response in fashion supply chains with dual information updating," Journal of Industrial and Management Optimization, vol. 2, no. 3, pp. 255-268, 2006.

[11] A. Bardakci and J. Whitelock, "Mass-customisation in marketing: the consumer perspective," Journal of Consumer Marketing, vol. 20, no. 5, pp. 463-479, 2003.

[12] N. Franke, M. Schreier, and U. Kaiser, "The "I designed it myself" effect in mass customization," Management Science, vol. 56, no. 1, pp. 125-140, 2010.

[13] A. Merle, J. L. Chandon, E. Roux, and F. Alizon, "Perceived value of the mass-customized product and mass customization experience for individual consumers," Production and Operations Management, vol. 19, no. 5, pp. 503-514, 2010.

[14] N. Liu, T. M. Choi, M. C. W. Yuen, and F. Ng, "An exploratory study on expert systems applications in fashion mass customization," in Proceedings of the 8th International Conference on Machine Learning and Cybernetics (ICMLC '09), pp. 36063610, Baoding, China, July 2009.

[15] G. Wan, "Internet based customer decision support systems for mass customization," International Journal of Management, vol. 17, no. 3, pp. 386-393, 2000.

[16] L. Peters and H. Saidin, "IT and the mass customization of services: the challenge of implementation," International Journal of Information Management, vol. 20, no. 2, pp. 103-119, 2000.

[17] P. R. Apeagyei and R. Otieno, "Usability of pattern customising technology in the achievement and testing of fit for mass customisation," Journal of Fashion Marketing and Management, vol. 11, no. 3, pp. 349-365, 2007. 
[18] T. M. Choi, P. S. Chow, and S. C. Liu, "Implementationof fashion ERP systems in China: case study of a fashion brand, review and future challenges," International Journal of Production Economics. In press.

[19] A. Kusiak and F. A. Salustri, "Computational intelligence in product design engineering: review and trends," IEEE Transactions on Systems, Man and Cybernetics C, vol. 37, no. 5, pp. 766778, 2007.

[20] J. Li and N. Huang, "Quality evaluation in flexible manufacturing systems: a markovian approach," Mathematical Problems in Engineering, vol. 2007, Article ID 57128, 24 pages, 2007.

[21] D. Liu, Z. Li, and M. C. Zhou, "Hybrid liveness-enforcing policy for generalized petri net models of flexible manufacturing systems," IEEE Transactions on Systems, Man, and Cybernetics: Systems, vol. 43, no. 1, pp. 85-97, 2013.

[22] H. Cavusoglu, H. Cavusoglu, and S. Raghunathan, "Selecting a customization strategy under competition: mass customization, targeted mass customization, and product proliferation," IEEE Transactions on Engineering Management, vol. 54, no. 1, pp. 12 28, 2007.

[23] H. Mendelson and A. K. Parlakturk, "Competitive customization," Manufacturing and Service Operations Management, vol. 10, no. 3, pp. 377-390, 2008.

[24] N. Takagoshi and N. Matsubayashi, "Customization competition between branded firms: continuous extension of product line from core product," European Journal of Operational Research, vol. 225, no. 2, pp. 337-352, 2013.

[25] C. H. Chiu, T. M. Choi, and D. Li, "Price wall or war: the pricing strategies for retailers," IEEE Transactions on Systems, Man, and Cybernetics A, vol. 39, no. 2, pp. 331-343, 2009.

[26] B. Ives and G. Piccoli, "Custom made apparel and individualized service at Lands' End," Communications of the Association For InFormation Systems, vol. 11, pp. 79-93, 2003.

[27] H. T. Yeung and T. M. Choi, "Mass customization in the Hong Kong apparel industry," Production Planning and Control, vol. 22, no. 3, pp. 298-307, 2011.

[28] N. Liu, T. M. Choi, M. C. W. Yuen, and F. Ng, "Optimal pricing, modularity and return policy under mass customization," IEEE Transactions on Systems, Man, and Cybernetics A, vol. 42, no. 3, pp. 604-614, 2012.

[29] T. Xiao, T. M. Choi, D. Yang, and T. C. E. Cheng, "Service commitment strategy and pricing decisions in retail supply chains with risk-averse players," Service Science, vol. 4, pp. 236252, 2012.

[30] C. Da Cunha, B. Agard, and A. Kusiak, "Design for cost: module-based mass customization," IEEE Transactions on Automation Science and Engineering, vol. 4, no. 3, pp. 350-359, 2007.

[31] J. H. Mikkola and O. Gassmann, "Managing modularity of product architectures: toward an integrated theory," IEEE Transactions on Engineering Management, vol. 50, no. 2, pp. 204-218, 2003.

[32] S. K. Mukhopadhyay and R. Setoputro, "Optimal return policy and modular design for build-to-order products," Journal of Operations Management, vol. 23, no. 5, pp. 496-506, 2005.

[33] J. D. Hess and G. E. Mayhew, "Modeling merchandise returns in direct marketing," Journal of Interactive Marketing, vol. 11, no. 2, pp. 20-35, 1997.

[34] T. M. Choi, "Optimal return service charging policy for fashion mass customization program," Service Science, vol. 5, no. 1, pp. 56-68, 2013.
[35] T. Volling and T. S. Spengler, "Modeling and simulation of order-driven planning policies in build-to-order automobile production," International Journal of Production Economics, vol. 131, no. 1, pp. 183-193, 2011.

[36] S. Chen and M. M. Tseng, "A Negotiation-Credit-Auction mechanism for procuring customized products," International Journal of Production Economics, vol. 127, no. 1, pp. 203-210, 2010.

[37] P. V. Ulrich, L. J. Anderson-Connel, and W. Wu, "Consumer codesign of apparel for mass customization," Journal of Fashion Marketing and Management, vol. 7, no. 4, pp. 398-412, 2003.

[38] S. L. Vargo and M. A. Akaka, "Value cocreation and service systems (re)formation: a service ecosystems view," Service Science, vol. 4, no. 3, pp. 207-217, 2012.

[39] A. Smedlund, "Value cocreation in service platform business models," Service Science, vol. 4, no. 1, pp. 79-88, 2012.

[40] F. S. Fogliatto, G. J. C. da Silveira, and D. Borenstein, "The mass customization decade: an updated review of the literature," International Journal of Production Economics, vol. 138, no. 1, pp. 14-25, 2012.

[41] X. J. Gu, G. N. Qi, Z. X. Yang, and G. J. Zheng, "Research of the optimization methods for mass customization (MC)," Journal of Materials Processing Technology, vol. 129, no. 1-3, pp. 507-512, 2002.

[42] M. M. Helms, M. Ahmadi, W. J. K. Jih, and L. P. Ettkin, "Technologies in support of mass customization strategy: exploring the linkages between e-commerce and knowledge management," Computers in Industry, vol. 59, no. 4, pp. 351-363, 2008.

[43] S. Bock, "Supporting offshoring and nearshoring decisions for mass customization manufacturing processes," European Journal of Operational Research, vol. 184, no. 2, pp. 490-508, 2008.

[44] A. Brun and M. Zorzini, "Evaluation of product customization strategies through modularization and postponement," International Journal of Production Economics, vol. 120, no. 1, pp. 205220, 2009.

[45] J. Yao and L. Liu, "Optimization analysis of supply chain scheduling in mass customization," International Journal of Production Economics, vol. 117, no. 1, pp. 197-211, 2009.

[46] R. Y. Zhong, Q. Y. Dai, T. Qu, G. J. Hu, and G. Q. Huang, "RFIDenabled real-time manufacturing execution system for masscustomization production," Robotics and Computer-Integrated Manufacturing, vol. 29, no. 2, pp. 283-292, 2013.

[47] T. M. Choi, N. Liu, F. Ng, and M. Yuen, "Mean-risk analysis of fashion mass customization programme with demand-return correlation," Working Paper, The Hong Kong Polytechnic University, 2012.

[48] H. M. Markowitz, Portfolio Selection: Efficient Diversification of Investments, Cowles Foundation for Research in Economics at Yale University, Monograph 16, John Wiley \& Sons, New York, NY, USA, 1959.

[49] C. H. Chiu, T. M. Choi, H. T. Yeung, and Y. Zhao, "Sales rebate contracts in fashion supply chains," Mathematical Problems in Engineering, vol. 2012, Article ID 908408, 19 pages, 2012.

[50] S. X. Xu, Q. Lu, and Z. Li, "Optimal modular production strategies under market uncertainty: a real options perspective," International Journal of Production Economics, vol. 139, no. 1, pp. 266-274, 2012.

[51] C. H. Chiu, T. M. Choi, and C. S. Tang, "Price, rebate, and returns supply contracts for coordinating supply chains with 
price-dependent demands," Production and Operations Management, vol. 20, no. 1, pp. 81-91, 2011.

[52] T. M. Choi, "Coordination and risk analysis of VMI supply chains with RFID technology," IEEE Transactions on Industrial Informatics, Vol, vol. 7, no. 3, pp. 497-504, 2011.

[53] T. M. Choi and C. H. Chiu, "Mean-downside-risk and meanvariance newsvendor models: implications for sustainable fashion retailers," International Journal of Production Economics, vol. 135, no. 2, pp. 552-560, 2012.

[54] T. M. Choi, C. H. Chiu, and P. L. Fu, "Periodic review multiperiod inventory control under a mean-variance optimization objective," IEEE Transactions on Systems, Man, and Cybernetics A, vol. 41, no. 4, pp. 678-682, 2011.

[55] Y. Wei and T. M. Choi, "Mean-variance analysis of supply chains under wholesale pricing and profit sharing schemes," European Journal of Operational Research, vol. 204, no. 2, pp. 255-262, 2010.

[56] C. H. Chiu, T. M. Choi, and X. Li, "Supply chain coordination with risk sensitive retailer under target sales rebate," Automatica, vol. 47, no. 8, pp. 1617-1625, 2011.

[57] B. Shen, T. M. Choi, Y. Wang, and C. K. Y. Lo, "The coordination of fashion supply chains with a risk averse supplier under the markdown money policy," IEEE Transactions on Systems, Man, and Cybernetics: Systems, vol. 43, no. 2, pp. 266-276, 2013. 


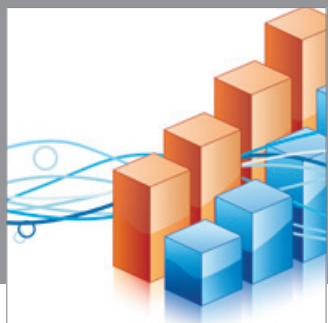

Advances in

Operations Research

mansans

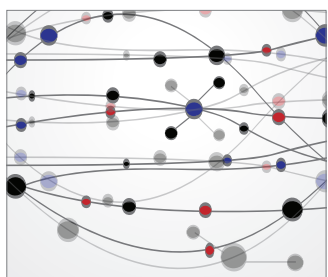

The Scientific World Journal
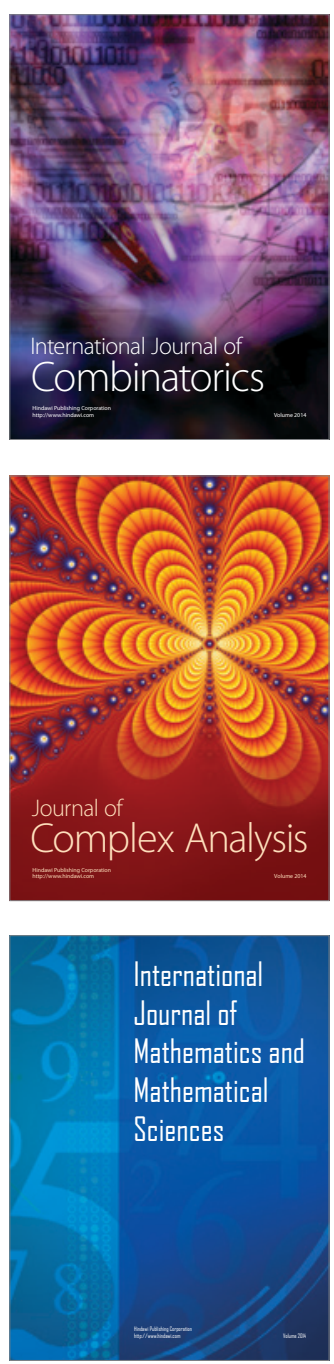
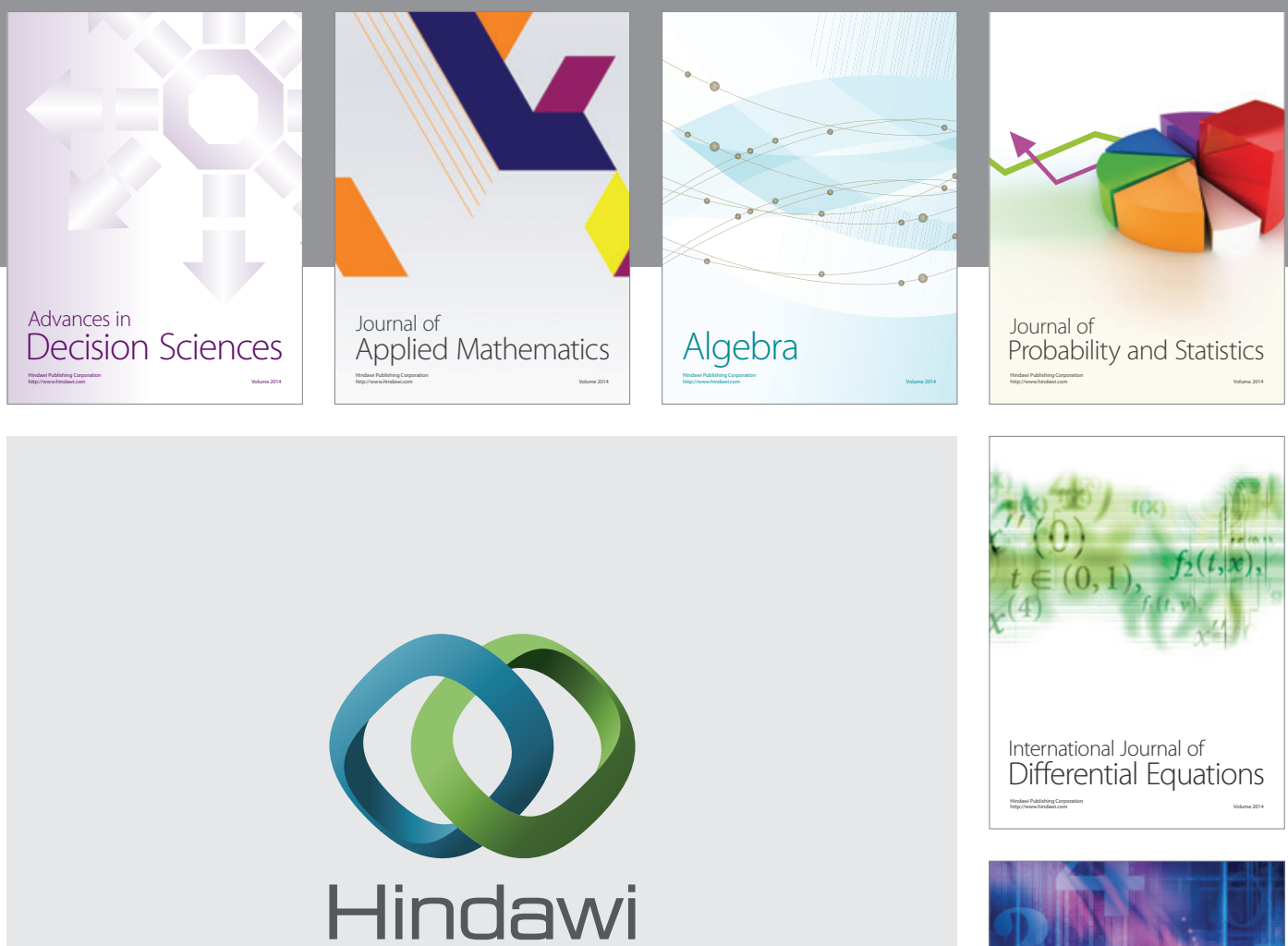

Submit your manuscripts at http://www.hindawi.com
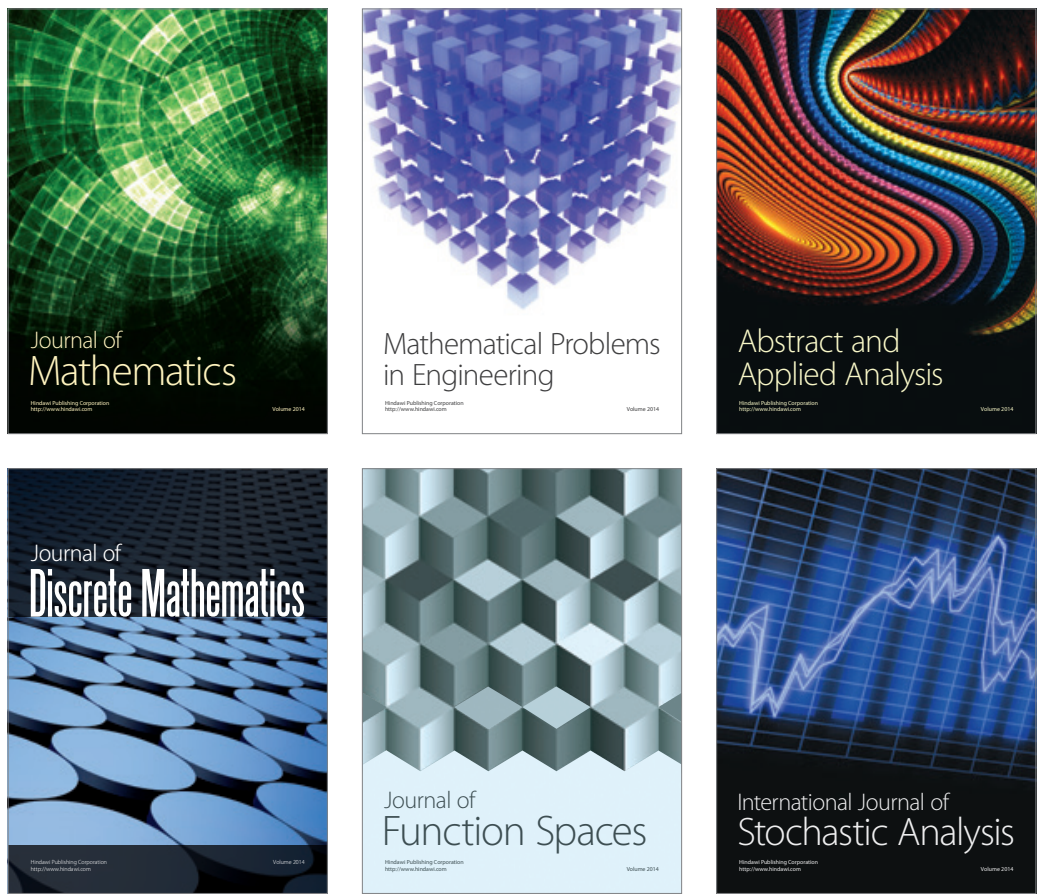

Journal of

Function Spaces

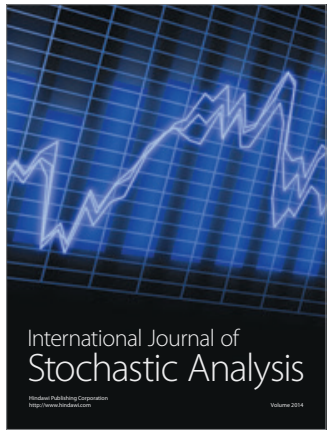

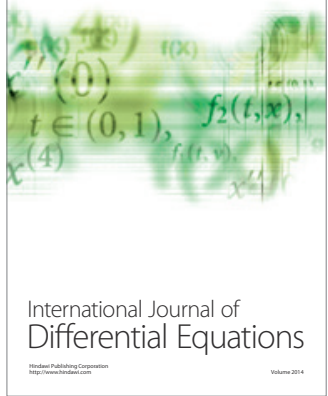
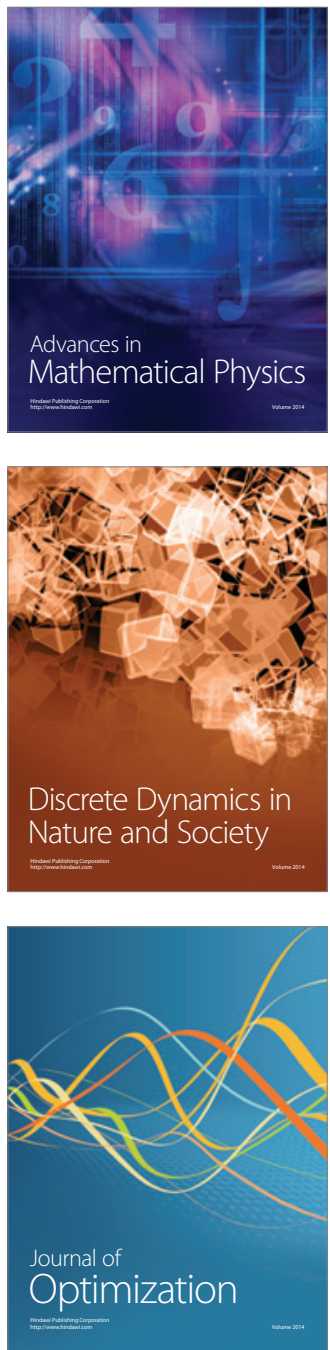